\title{
Bargaining with endogenous disagreement: the extended Kalai-Smorodinsky solution
}

Citation for published version (APA):

Bozbay, I., Dietrich, F. K., \& Peters, H. J. M. (2010). Bargaining with endogenous disagreement: the extended Kalai-Smorodinsky solution. METEOR, Maastricht University School of Business and Economics. METEOR Research Memorandum No. 008 https://doi.org/10.26481/umamet.2010008

Document status and date:

Published: 01/01/2010

DOI:

10.26481/umamet.2010008

Document Version:

Publisher's PDF, also known as Version of record

\section{Please check the document version of this publication:}

- A submitted manuscript is the version of the article upon submission and before peer-review. There can be important differences between the submitted version and the official published version of record.

People interested in the research are advised to contact the author for the final version of the publication, or visit the DOI to the publisher's website.

- The final author version and the galley proof are versions of the publication after peer review.

- The final published version features the final layout of the paper including the volume, issue and page numbers.

Link to publication

\footnotetext{
General rights rights.

- You may freely distribute the URL identifying the publication in the public portal. please follow below link for the End User Agreement:

www.umlib.nl/taverne-license

Take down policy

If you believe that this document breaches copyright please contact us at:

repository@maastrichtuniversity.nl

providing details and we will investigate your claim.
}

Copyright and moral rights for the publications made accessible in the public portal are retained by the authors and/or other copyright owners and it is a condition of accessing publications that users recognise and abide by the legal requirements associated with these

- Users may download and print one copy of any publication from the public portal for the purpose of private study or research.

- You may not further distribute the material or use it for any profit-making activity or commercial gain

If the publication is distributed under the terms of Article $25 \mathrm{fa}$ of the Dutch Copyright Act, indicated by the "Taverne" license above, 
I rem Bozbay, Franz Dietrich, Hans Peters

Bargaining with endogenous disagreement: the extended Kalai-Smorodinsky solution

$\mathrm{RM} / 10 / 008$

\section{METEOR}

Maastricht University School of Business and Economics

Maastricht Research School of Economics

of Technology and Organization

P.O. Box 616

NL - 6200 MD Maastricht

The Netherlands 


\title{
Bargaining with endogenous disagreement: the extended Kalai-Smorodinsky solution*
}

\author{
Irem Bozbay ${ }^{\dagger} \quad$ Franz Dietrich ${ }^{\ddagger} \quad$ Hans Peters $^{\dagger}$
}

March 2010

\begin{abstract}
Following Vartiainen (2007) we consider bargaining problems in which no exogenous disagreement outcome is given. A bargaining solution assigns a pair of outcomes to such a problem, namely a compromise outcome as well as a disagreement outcome: the interpretation is that the latter results if the compromise outcome is not accepted. For this framework we propose and study an extension of the classical Kalai-Smorodinsky bargaining solution. We present a characterization with an axiom of Independence of Non-Utopia Information as the main condition.
\end{abstract}

\section{JEL-classification: C78, D74}

Keywords: Axiomatic bargaining, endogenous disagreement outcome, extended Kalai-Smorodinsky solution

\section{Introduction}

In the bargaining problem of Nash (1950) each player can unilaterally enforce the disagreement outcome if negotiations fail. In some cases, however, it may not be clear what the disagreement outcome is or whether the players can, or want to, enforce it if agreement is not reached. In the classical example of employer-union wage negotiations the union can call out a strike if it is not satisfied with the wage offered by the employer. But how long should the strike last? What will be its consequences? Will all workers join? Are there perhaps different and better ways to put pressure on management? Also, which outcome can the employer enforce, if any, in case no agreement is reached?

In this paper, following Vartiainen (2007), we assume that the disagreement outcome is determined endogenously, namely by the bargaining solution. A

\footnotetext{
*This paper owes much to Valkengoed (2006).

$\dagger$ Department of Quantitative Economics, Maastricht University.

Email: I.Bozbay@maastrichtuniversity.nl, H.Peters@maastrichtuniversity.nl.

${ }^{\ddagger}$ London School of Economics and Department of Quantitative Economics, Maastricht University. Email: F.Dietrich@lse.ac.uk.
} 
natural interpretation of this approach is to assume that there is an arbitrator who proposes a pair of outcomes, namely a compromise outcome and a disagreement outcome. If both players accept the compromise outcome then this is implemented; otherwise, the disagreement outcome results.

Within this framework, Vartiainen (2007) proposes and axiomatically characterizes a bargaining solution which extends the classical Nash bargaining solution for bargaining problems with fixed disagreement point. That solution maximizes the Nash product, i.e., the product of the gains of the players from the compromise outcome over the disagreement outcome.

By contrast, the solution proposed in our paper depends explicitly on the utopia point and extends the solution of Raiffa (1953) and Kalai-Smorodinsky (1975) for classical bargaining problems. This extension works as follows. First, the assigned compromise point is indeed the classical Kalai-Smorodinsky (KS) outcome for the assigned disagreement outcome. That is, it is the Pareto optimal point on the straight line joining this disagreement outcome and its associated utopia point. Second, the assigned disagreement outcome is the point on the straight line joining the assigned compromise point and the associated 'antiutopia point', obtained by taking the minimum utilities of the players below the compromise point; it is, thus, a 'converse' KS outcome. We present a characterization of this solution in which, indeed, the crucial axiom is a condition of Independence of Non-Utopia information.

Another extension of the Kalai-Smorodinsky solution to bargaining problems without fixed disagreement point is proposed in Vartiainen (2002), but this solution is quite different from our extension.

The framework in our paper and in Vartiainen (2007) is similar to the one in Thomson (1981), who also considers bargaining problems without disagreement point. So, a bargaining problem is defined merely as a utility-possibility set. Thomson introduces the notion of reference point as a function of the bargaining problem. The key difference to the classical disagreement point is that no player can unilaterally enforce the reference point. It may thus serve, rather, as a hypothetical outcome to which the players compare proposals made during negotiations. In situations where an arbitrator, or a mediator, makes choices for the players (cf. Luce and Raiffa, 1957), the reference point can be the outcome to be implemented when players do not agree on the solution proposed by the arbitrator, or a comparison point to which the players or the arbitrator compare any bargaining outcome. The first is the case when arbitration is binding: the players either unanimously accept the proposed solution, or the reference point results.

In the next section we show that the extended KS-solution is non-empty valued and we characterize the domain of bargaining problems for which it is single-valued. In Section 3 we present an axiomatic characterization of the solution on domains where it is single-valued.

Notation For $x, y \in \mathbb{R}^{2}, x>y$ means $x_{i}>y_{i}$ and $x \geqslant y$ means $x_{i} \geqslant y_{i}$ for $i=1,2$. Similarly for $<$ and $\leqslant$. By $[x, y]$ we denote the line segment with endpoints $x$ and $y$. The cardinality of a set $X \subseteq \mathbb{R}^{2}$ is denoted by $|X|$. For 
$a, x \in \mathbb{R}^{2}, a x:=\left(a_{1} x_{1}, a_{2} x_{2}\right), a X:=\{a x \mid x \in X\}$, and $a+X:=\{a+x \mid x \in X\}$. The set $(-1,-1) X$ is also denoted by $-X$. By $\mathbb{R}_{+}^{2}$ we denote the (strictly) positive quadrant of $\mathbb{R}^{2}$.

\section{Bargaining problems and the extended Kalai- Smorodinsky solution}

A bargaining problem $U$ is a compact and convex subset of $\mathbb{R}^{2}$ such that $x>y$ for some $x, y \in U$. Elements of $U$ are called outcomes and represent the utilities of two players. By $\mathcal{U}$ we denote the set of all bargaining problems. A classical bargaining problem is a pair $(U, d)$, where $U \in \mathcal{U}$ and $d \in U$; the outcome $d$ is called the disagreement outcome, and it results if the players do not reach agreement. By $\mathcal{B}$ we denote the set of all classical bargaining problems.

A bargaining solution or, briefly, a solution is a correspondence $f: \mathcal{U} \rightarrow$ $\mathbb{R}^{2} \times \mathbb{R}^{2}$ such that $s, r \in U$ and $s \neq r$ for all $U \in \mathcal{U}$ and $(s, r) \in f(U)$. For a pair $(s, r) \in f(U)$, we call $s$ the compromise outcome and $r$ the disagreement outcome. The interpretation is that either the players reach the compromise $s$ or, otherwise, $r$ results - where these points are possibly proposed by a mediator. A classical bargaining solution is a map $F: \mathcal{B} \rightarrow \mathbb{R}^{2}$ with $F(U, d) \in U$ for all $(U, d) \in \mathcal{B}$.

For a bargaining problem $U \in \mathcal{U}$, the Pareto optimal set is the set

$$
P(U):=\{x \in U \mid \text { for all } y \in U, y \geqslant x \text { implies } y=x\}
$$

and the anti-Pareto optimal set is the set

$$
A P(U):=\{x \in U \mid \text { for all } y \in U, y \leqslant x \text { implies } y=x\} .
$$

For $(U, d) \in \mathcal{B}$ the utopia point is the point

$$
u(U, d):=\left(\max \left\{x_{1} \mid x \in U, x \geqslant d\right\}, \max \left\{x_{2} \mid x \in U, x \geqslant d\right\}\right) .
$$

In this paper we focus on a particular solution, which extends the classical Kalai-Smorodinsky bargaining solution (Raiffa, 1953; Kalai and Smorodinsky, $1975)$. The latter solution assigns to each classical bargaining problem $(U, d)$ the unique point $K S(U, d) \in P(U)$ on the straight line through $d$ and $u(U, d)$. The extended Kalai-Smorodinsky solution is the correspondence $k: \mathcal{U} \rightarrow \mathbb{R}^{2} \times \mathbb{R}^{2}$ defined by

$$
(s, r) \in k(U) \Leftrightarrow s=K S(U, r), r=-K S(-U,-s) \text { and } s \neq r
$$

for all $U \in \mathcal{U}$ and $s, r \in U$. Thus, $(s, r) \in k(U)$ exactly if the following three conditions are satisfied: (i) $s \neq r$; (ii) $s$ is the classical Kalai-Smorodinsky outcome when $r$ is viewed as the disagreement outcome; and (iii) $r$ results similarly from $s$ when we reverse the problem or, equivalently, $r$ is the unique point in $A P(U)$ on the straight line through $s$ and the anti-utopia point

$$
a(U, s):=\left(\min \left\{x_{1} \mid x \in U, x \leqslant s\right\}, \min \left\{x_{2} \mid x \in U, x \leqslant s\right\}\right) .
$$


Our first result is that the extended Kalai-Smorodinsky solution is nonempty valued. The proof is based on an elementary fixed point argument, slightly complicated by the fact that the Pareto and anti-Pareto optimal sets of a bargaining problem $U$ may have one or both endpoints in common. Clearly, in that case, by definition of $k$-in particular the condition $s \neq r$ - such an endpoint cannot be the solution outcome.

Theorem $2.1 k(U) \neq \emptyset$ for all $U \in \mathcal{U}$.

Proof. Let $U \in \mathcal{U}$. Then $A P(U)$ is the graph of a strictly decreasing convex function $g$ on an interval $[\alpha, \beta]$ with $(\alpha, g(\alpha))$ and $(\beta, g(\beta))$ the points of $A P(U)$ with minimal and maximal first coordinates, respectively. If $\alpha=\beta$ (so that $A P(U)$ consists of a unique outcome $)$ then $\{(K S(U,(\alpha, g(\alpha))),(\alpha, g(\alpha)))\}=$ $k(U)$ and we are done. From now on we assume $\alpha<\beta$. Define the function $\varphi:[\alpha, \beta] \rightarrow[\alpha, \beta]$ by $\varphi(\gamma)=-K S(-U,-K S(U,(\gamma, g(\gamma))))$. Observe that if $\varphi\left(\gamma^{*}\right)=\gamma^{*}$ for some $\gamma^{*} \in[\alpha, \beta]$ and $K S\left(U,\left(\gamma^{*}, g\left(\gamma^{*}\right)\right)\right) \neq\left(\gamma^{*}, g\left(\gamma^{*}\right)\right)$ then $\left(K S\left(U,\left(\gamma^{*}, g\left(\gamma^{*}\right)\right)\right),\left(\gamma^{*}, g\left(\gamma^{*}\right)\right)\right) \in k(U)$.

Of course, $\varphi(\alpha) \geqslant \alpha$ and $\varphi(\beta) \leqslant \beta$. Suppose that $(\alpha, g(\alpha)) \in P(U)$. Then $\varphi(\alpha)=\alpha$, but $(K S(U,(\alpha, g(\alpha))),(\alpha, g(\alpha))) \notin k(U)$ since $K S(U,(\alpha, g(\alpha)))=$ $(\alpha, g(\alpha))$. Below, however, we will prove:

$$
\text { There is } \varepsilon_{1}>0 \text { with } \varphi(\gamma)>\gamma \text { for all } \gamma \in\left(\alpha, \alpha+\varepsilon_{1}\right] \text {. }
$$

Similarly, if $(\beta, g(\beta)) \in P(U)$ we have:

There is $\varepsilon_{2}>0$ with $\varphi(\gamma)<\gamma$ for all $\gamma \in\left[\beta-\varepsilon_{2}, \beta\right)$.

Clearly, we can then take $\varepsilon_{1}$ and $\varepsilon_{2}$ in (1) and (2) such that $\alpha+\varepsilon_{1}<\beta-\varepsilon_{2}$. Now define the interval $\left[\alpha^{\prime}, \beta^{\prime}\right]$ by $\alpha^{\prime}=\alpha$ if $(\alpha, g(\alpha)) \notin P(U)$ and $\alpha^{\prime}=\alpha+$ $\varepsilon_{1}$ if $(\alpha, g(\alpha)) \in P(U)$, and $\beta^{\prime}=\beta$ if $(\beta, g(\beta)) \notin P(U)$ and $\beta^{\prime}=\beta-\varepsilon_{2}$ if $(\beta, g(\beta)) \in P(U)$. Then, since $\varphi$ is continuous, the intermediate value theorem implies that in all cases there is a point $\gamma^{*} \in\left[\alpha^{\prime}, \beta^{\prime}\right]$ with $\varphi\left(\gamma^{*}\right)=\gamma^{*}$ and $K S\left(U,\left(\gamma^{*}, g\left(\gamma^{*}\right)\right)\right) \neq\left(\gamma^{*}, g\left(\gamma^{*}\right)\right)$ and, thus, $k(U) \neq \emptyset$.

We are left to prove (1) and (2). We only show (1), the proof of (2) is analogous. So suppose $z:=(\alpha, g(\alpha)) \in P(U)$. See Figure 1 for an illustration of the remainder of the proof.

Let $m$ and $\ell$ be the supporting lines of $U$ at $z$ as drawn in Figure 1. (That is, $m$ is the limit of supporting lines at $P(U)$ and $\ell$ is the limit of supporting lines at $A P(U)$.) Let $\mu$ be the absolute value of the slope of $m$ and let $\lambda$ be the absolute value of the slope of $\ell$. Then $\lambda>\mu$.

For $x \in A P(U) \backslash P(U)$ let $\sigma(x)$ denote the slope of the straight line through $x$ and $u(U, x)$. Let $c[x]$ denote the line segment with endpoints $\left(x_{1}, u_{2}(U, x)\right)$ and $\left(u_{1}(U, x), x_{2}\right)$. Then $\sigma(x)$ is equal to the absolute value of the slope of $c[x]$. Therefore, $\sigma(x)$ converges to $\mu$ if $x \in A P(U)$ converges to $z$.

For $y \in P(U) \backslash A P(U)$ let $\tau(y)$ denote the slope of the straight line through $y$ and $a(U, y)$. Let $c[y]$ denote the line segment with endpoints $\left(y_{1}, a_{2}(U, y)\right)$ and $\left(a_{1}(U, y), y_{2}\right)$. Then $\tau(y)$ is equal to the absolute value of the slope of $c[y]$. Therefore, $\tau(y)$ converges to $\lambda$ if $y \in P(U)$ converges to $z$. 


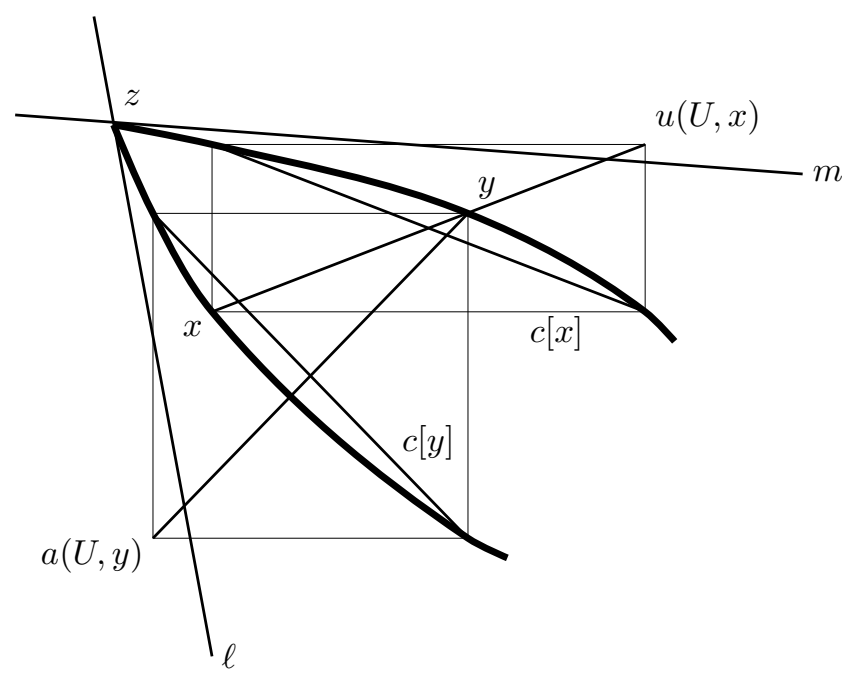

Figure 1: Illustrating the proof of (1)

We conclude that $\tau(y)>\sigma(x)$ for $y \in P(U)$ and $x \in A P(U)$ close to $z$. This implies the existence of an $\varepsilon_{1}$ as in (1).

Observe that $k$ does not have to assign a unique pair of outcomes to a bargaining problem. For instance, let $U$ be the convex hull of the points $(6,0)$, $(8,0),(0,6)$, and $(0,8)$. Then it is not difficult to check that

$$
k(U)=\left\{\left(\left(s_{1}, s_{2}\right),\left(r_{1}, r_{2}\right)\right) \mid 2 \leqslant s_{1} \leqslant 6, r_{1}=s_{1}-1, s_{1}+s_{2}=8, r_{1}+r_{2}=6\right\} .
$$

In this example the Pareto optimal and anti-Pareto optimal sets are parallel line segments. In fact, a sufficient but not necessary condition for $k$ to assign a unique pair of outcomes to a problem $U$ is that $P(U)$ and $A P(U)$ do not contain parallel line segments. Theorem 2.2 below provides an exact description of the class of all bargaining problems on which $k$ is unique. We first introduce some additional terminology.

For $x \neq y$ and $x^{\prime} \neq y^{\prime}$ the line segments $[x, y]$ and $\left[x^{\prime}, y^{\prime}\right]$ are parallel if the straight lines $\ell$ and $\ell^{\prime}$ containing these line segments are parallel. In that case, the vertical distance between $[x, y]$ and $\left[x^{\prime}, y^{\prime}\right]$ is the number $v=\left|z_{2}-z_{2}^{\prime}\right|$ for (any) $z \in \ell$ and $z^{\prime} \in \ell^{\prime}$ with $z_{1}=z_{1}^{\prime} ; v$ is infinite if $\ell$ and $\ell^{\prime}$ are vertical. Similarly, the horizontal distance between $[x, y]$ and $\left[x^{\prime}, y^{\prime}\right]$ is the number $h=\left|z_{1}-z_{1}^{\prime}\right|$ for (any) $z \in \ell$ and $z^{\prime} \in \ell^{\prime}$ with $z_{2}=z_{2}^{\prime} ; h$ is infinite if $\ell$ and $\ell^{\prime}$ are horizontal.

Now let $\mathcal{D}_{k}$ denote the set of bargaining problems $U$ with $|k(U)|=1$.

Theorem 2.2 Let $U \in \mathcal{U}$. Then $U \in \mathcal{D}_{k}$ if and only if there are no parallel line segments $[\bar{x}, \underline{x}] \subseteq A P(U)]$ and $[\bar{y}, \underline{y}] \subseteq P(U)$ with $\bar{x}_{1}<\underline{x}_{1}$ and $\bar{y}_{1}<\underline{y}_{1}$ and such that the vertical distance $v$ and horizontal distance $h$ between these line segments satisfy the following conditions:

(i) $\frac{1}{2} v=\bar{y}_{2}-\bar{x}_{2}=\underline{y}_{2}-\underline{x}_{2}$, 
(ii) the lengths of $[\bar{x}, \underline{x}]$ and $[\bar{y}, \underline{y}]$ both exceed $\sqrt{h^{2}+v^{2}}$.

A proof of this theorem is provided in the appendix. See also Figure 3 (in the appendix) for an illustration. The theorem implies that we do not lose much generality if we restrict attention to domains of bargaining problems within $\mathcal{D}_{k}$. We conclude this section with a corollary to Theorem 2.2, listing some domains on which $k$ is single-valued.

Corollary 2.3 The extended Kalai-Smorodinsky solution $k$ is single-valued on each of the following domains:

(a) $\{U \in \mathcal{U} \mid U$ is strictly convex $\}$.

(b) $\{U \in \mathcal{U} \mid A P(U)$ or $P(U)$ contains no line segment $\}$.

(c) $\left\{U \in \mathcal{U} \mid\right.$ there are no parallel line segments $S$ and $S^{\prime}$ with $S \subseteq A P(U)$ and $\left.S^{\prime} \subseteq P(U)\right\}$.

\section{An axiomatic characterization of the extended Kalai-Smorodinsky solution}

In this section we give an axiomatic characterization of the extended KalaiSmorodinsky solution $k$ on domains which are minimally rich (defined below, see also Remark 3.4) and on which $k$ is single-valued.

A bargaining problem $U^{\prime} \in \mathcal{U}$ is a positive affine transformation of a bargaining problem $U \in \mathcal{U}$ if there are $a \in \mathbb{R}_{+}^{2}$ and $b \in \mathbb{R}^{2}$ such that $U^{\prime}=a U+b$. A bargaining problem $U \in \mathcal{U}$ is symmetric if $U=\left\{\left(x_{2}, x_{1}\right) \mid\left(x_{1}, x_{2}\right) \in U\right\}$. Let $\mathcal{D}_{k}^{s}$ denote the set of all symmetric polytopes ${ }^{1}$ in $\mathcal{D}_{k}$ and their positive affine transformations. A domain of bargaining problems $\mathcal{D} \subseteq \mathcal{U}$ is minimally rich if $\mathcal{D}_{k}^{s} \subseteq \mathcal{D}$.

We formulate our conditions for a solution $f$ defined on a minimally rich domain $\mathcal{D} \subseteq \mathcal{U}$ with $|f(U)|=1$ for all $U \in \mathcal{D}$. Instead of $f(U)=\{(s, r)\}$ we write $f(U)=(s, r)$ and regard $f$ as a function rather than a correspondence.

The first condition is an extended version of the usual Pareto optimality condition.

Extended Pareto Optimality (EPO): For each $U \in \mathcal{D}, f(U) \in P(U) \times A P(U)$.

In particular from a normative view point it is natural to require Pareto optimality of the compromise outcome. Requiring anti-Pareto optimality of the disagreement outcome reflects that we wish this outcome to be as severe as possible in order to induce acceptance of the compromise outcome.

The following two conditions are standard in classical axiomatic bargaining theory. They have similar justifications in the present model.

\footnotetext{
${ }^{1} \mathrm{~A}$ polytope is the convex hull of finitely many points.
} 
Symmetry (SYM): For each symmetric $U \in \mathcal{D}$, if $f(U)=(s, r)$ then $s_{1}=s_{2}$ and $r_{1}=r_{2}$.

Scale Invariance (SI): For all $U \in \mathcal{D}$ and $a \in \mathbb{R}_{+}^{2}, b \in \mathbb{R}^{2}$ with $a U+b \in \mathcal{D}$, if $f(U)=(s, r)$ then $f(a U+b)=(a s+b, a r+b)$.

The fourth and final condition extends and modifies similar conditions used in characterizations of the classical Kalai-Smorodinsky solution.

Independence of Non-Utopia Information (INU): For all $U, V \in \mathcal{D}$, if $f(V)=$ $(s, r) \in P(V) \times A P(V), f(V) \in P(U) \times A P(U), u(U, r)=u(V, r)$ and $a(U, s)=$ $a(V, s)$, then $f(U)=(s, r)$.

This condition says that if $f(V)=(s, r) \in P(V) \times A P(V)$ and we consider a problem $U$ such that $s$ and $r$ are still Pareto and anti-Pareto optimal in $U$ and also the associated utopia and anti-utopia points do not change, then the solution does not change: $f(U)=(s, r)$ as well. Together with the other three axioms this condition uniquely characterizes the extended Kalai-Smorodinsky solution on any minimally rich subdomain of $\mathcal{D}_{k}$.

Theorem 3.1 Let $\mathcal{D} \subseteq \mathcal{D}_{k}$ be minimally rich and let $f: \mathcal{D} \rightarrow \mathbb{R}^{2} \times \mathbb{R}^{2}$ be a solution satisfying $|f(U)|=1$ for all $U \in \mathcal{D}$. Then the following two statements are equivalent:

(a) $f$ satisfies EPO, SYM, SI, and INU.

(b) $f(U)=k(U)$ for all $U \in \mathcal{D}$.

Proof. Clearly, $k$ satisfies the four conditions in (a) on the domain $\mathcal{D} \subseteq \mathcal{D}_{k}$. We now prove the converse implication $(a) \Rightarrow(b)$.

Suppose $f$ satisfies the four conditions and let $U \in \mathcal{D}$. We have to prove that $f(U)=k(U)$. Let $k(U)=(s, r) \in P(U) \times A P(U)$. Then $s>r$ (this follows from the requirement that there must be $x, y \in U$ with $x>y$ ). Let $V$ be the convex hull of the six points $s, r,\left(s_{1}, a_{2}(U, s)\right),\left(a_{1}(U, s), s_{2}\right),\left(u_{1}(U, r), r_{2}\right)$, and $\left(r_{1}, u_{2}(U, r)\right)$. We will prove that $V \in \mathcal{D}$ and $f(V)=(s, r)$. This will conclude the proof of the theorem, since by INU, $f(V)=(s, r)$ implies $f(U)=(s, r)$ and, thus, $f(U)=k(U)$.

Consider the positive affine transformation

$$
\left(x_{1}, x_{2}\right) \mapsto\left(\varphi_{1}\left(x_{1}\right), \varphi_{2}\left(x_{2}\right)\right):=\left(\frac{x_{1}-r_{1}}{s_{1}-r_{1}}, \frac{x_{2}-r_{2}}{s_{2}-r_{2}}\right)
$$

which maps $r$ to $(0,0), s$ to $(1,1)$, and $V$ to some set $V^{\prime}$. Then it is not hard to check that $V^{\prime}$ is a symmetric polytope, and it is sufficient to prove that $V^{\prime} \in \mathcal{D}_{k}$ : for this implies $V \in \mathcal{D}$ by minimal richness of $\mathcal{D}$; and by SYM and EPO, we have $f\left(V^{\prime}\right)=((1,1),(0,0))$ and thus, by SI, $f(V)=(s, r)$.

We are left to prove that $V^{\prime} \in \mathcal{D}_{k}$, i.e., that $\left|k\left(V^{\prime}\right)\right|=1$. Consider Figure 2 with notations as there. For $k\left(V^{\prime}\right)$ to be non-unique there are, in view of Theorem 2.2, two possible cases to examine: (1) $a$ is parallel to $d$ and (2) $a$ 


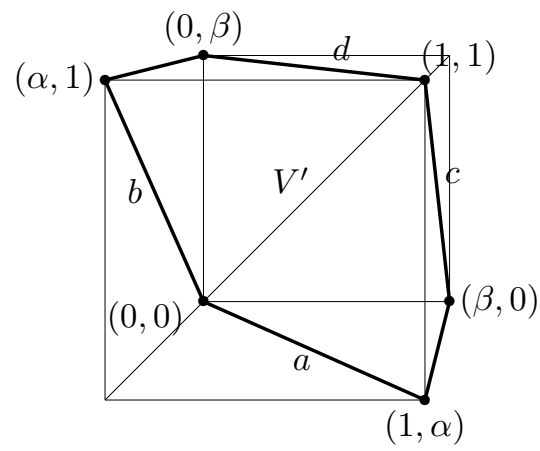

Figure 2: Illustrating the proof of Theorem 3.1. The letters $a, b, c, d$ denote line segments, $\alpha=\varphi_{1}\left(a_{1}(U, s)\right)=\varphi_{2}\left(a_{2}(U, s)\right)$, and $\beta=\varphi_{1}\left(u_{1}(U, r)\right)=\varphi_{2}\left(u_{2}(U, r)\right)$

is parallel to $c$. (The cases involving $b$ are analogous.) In case (1) we must have $\beta=1-\alpha>1$. Denote the vertical and horizontal distances between $a$ and $d$ by $v$ and $h$, then the length of $a$ is equal to $\sqrt{1+\alpha^{2}}$ whereas $\sqrt{v^{2}+h^{2}}>$ $\sqrt{\beta^{2}+\beta^{2}}>\sqrt{1+\alpha^{2}}$, so that $a$ does not satisfy condition (ii) in Theorem 2.2. In case (2) we must have $\beta=2$ and $\alpha=-1$. In particular, $A P\left(V^{\prime}\right)$ is the line segment $[(-1,1),(1,-1)]$ and $P\left(V^{\prime}\right)$ is the line segment $[(0,2),(2,0)]$, so that again condition (ii) in Theorem 2.2 is violated.

We conclude with a few remarks.

Remark 3.2 The characterization in Theorem 3.1 is tight, at least on the domain $\mathcal{D}_{k}$. The following examples serve as proofs.

(1) For $U \in \mathcal{U}$ let $u(U)=\left(\max \left\{x_{1} \mid x \in U\right\}, \max \left\{x_{2} \mid x \in U\right\}\right)$ and $a(U)=\left(\min \left\{x_{1} \mid x \in U\right\}, \min \left\{x_{2} \mid x \in U\right\}\right)$ be the global utopia and antiutopia points of $U$. Define the solution $f^{1}$ by $f^{1}(U)=(s, r)$ such that $s$ is the Nash bargaining solution outcome of $(U, a(U))$ and $-r$ is the Nash bargaining solution outcome of $(-U,-u(U))$. This solution satisfies EPO, SYM, and SI, but not INU on the domain $\mathcal{D}_{k}$.

(2) Define the solution $f^{2}$ on $\mathcal{D}_{k}$ by $f^{2}(U)=\left(\frac{3}{4} s+\frac{1}{4} r, \frac{1}{4} s+\frac{3}{4} r\right)$, where $(s, r)=k(U)$. This solution satisfies SYM, SI, and (trivially) INU, but not EPO.

(3) Define the solution $f^{3}$ in the same way as $k$ but now based on a nonsymmetric version of the KS-solution (cf. Peters and Tijs, 1985). Such a solution satisfies EPO, SI, and INU, but not SYM.

(4) Define the solution $f^{4}$ by $f^{4}(T)=\left(\left(3, \frac{1}{2}\right),(0,0),\right)$ where $T$ is the convex hull of $(0,0),(4,0)$, and $(0,2)$; and by $f^{4}(U)=k(U)$ for all $U \in \mathcal{D}_{k}$ with $U \neq T$. Then $f^{4}$ satisfies EPO, SYM, INU, but not SI.

Remark 3.3 A partial characterization of the extended Kalai-Smorodinsky solution on the whole domain $\mathcal{U}$ is provided in Valkengoed (2006), at the expense 
of rather technical conditions. As already mentioned, restricting ourselves to the domain $\mathcal{D}_{k}$ is without much loss of generality.

Remark 3.4 Variations on the characterization of $k$ can be obtained by imposing different conditions of 'minimal richness'. For instance, Theorem 3.1 would still hold - with a slight modification of the proof - on some subdomains of $\mathcal{D}_{k}$ that contain all strictly convex bargaining problems.

\section{References}

Kalai E, Smorodinsky M (1975) Other solutions to Nash's bargaining problem. Econometrica 43, 513-518

Luce D, Raiffa H (1957) Games and Decisions. Wiley, New York

Nash JF (1950) The bargaining problem. Econometrica 28, 155-162

Peters H, Tijs S (1985) Characterization of all individually monotonic bargaining solutions. International Journal of Game Theory 14, 219-228

Raiffa H (1953) Arbitration schemes for generalized two-person games. Contributions to the Theory of Games II. Ed. by Kuhn HW, Tucker AW. Annals of Mathematics Studies 28, 361-387. Princeton University Press, Princeton

Thomson W (1981) A class of solutions to the bargaining problem. Journal of Economic Theory 25, 431-442

Valkengoed J (2006) A monotone solution for bargaining problems with endogenous disagreement point. Master thesis, Maastricht University

Vartiainen H (2002) Bargaining without disagreement. Yrjö Jahnsson Foundation, Helsinki

Vartiainen H (2007) Collective choice with endogenous reference outcome. Games and Economic Behavior 58, 172-180

\section{A Appendix: proof of Theorem 2.2}

For the only-if part, suppose that $[\bar{x}, \underline{x}] \subseteq A P(U)]$ and $[\bar{y}, \underline{y}] \subseteq P(U)$ are as in the Theorem. Let $\bar{r} \in[\bar{x}, \underline{x}]$ with $\bar{r}_{1}=\bar{y}_{1}$ and $\underline{r} \in[\bar{x}, \underline{x}]$ with $\underline{r}_{2}=\underline{y}_{2}$. For each $r \in[\bar{r}, \underline{r}]$ let $s(r) \in[\bar{y}, y]$ with $s(r)_{2}-r_{2}=v / 2$. Then it is straightforward to check that $(s(r), r) \in \bar{k}(U)$ for each $r \in[\bar{r}, \underline{r}]$. Thus, $U \notin \mathcal{D}_{k}$. See Figure 3 for an illustration.

We now prove the if-part. Assume $U \notin \mathcal{D}_{k}$, i.e. $|k(U)|>1$. We will construct $[\bar{x}, \underline{x}] \subseteq A P(U)]$ and $[\bar{y}, \underline{y}] \subseteq P(U)$ as in the theorem.

For any $x \in A P(U)$ let $\sigma(x)$ denote the slope of the straight line through $x$ and $u(U, x)$ (as in the proof of Theorem 2.1). Since $\sigma(x)$ is equal to the absolute 


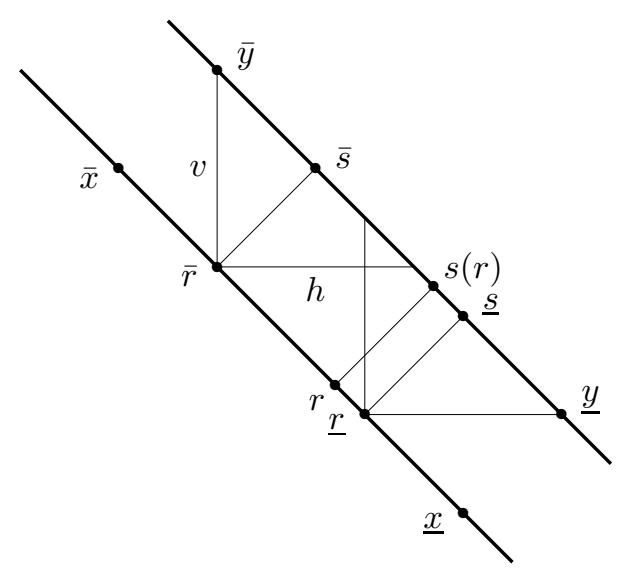

Figure 3: Illustrating the proof of Theorem 2.2

value of the slope of the line segment $c[x]$ connecting the points $\left(x_{1}, u_{2}(U, x)\right)$ and $\left(u_{1}(U, x), x_{2}\right)$, and the absolute values of these slopes weakly increase if $x_{1}$ increases - the line segments $c[x]$ are chords of the weakly decreasing concave function the graph of which contains the Pareto optimal set of $U$ - we have that $\sigma(x)$ weakly increases if $x_{1}$ increases.

Similarly, for any $y \in P(U)$ let $\tau(y)$ denote the slope of the straight line through $y$ and $a(U, y)$ (again as in the proof of Theorem 2.1). Then by an analogous argument $\tau(y)$ weakly increases if $y_{1}$ decreases.

Let $(\bar{s}, \bar{r})$ and $(\underline{s}, \underline{r})$ be the elements of $k(U)$ with maximal and minimal second coordinates, respectively. By definition of $k$ we have $\tau(s)=\sigma(r)$ for all $(s, r) \in k(U)$. Therefore, by $(*)$ and $(* *)$ we must have $\sigma(x)=\tau(y)$ for all $x \in A P(U)$ with $\bar{r}_{1} \leqslant x_{1} \leqslant \underline{r}_{1}$ and all $y \in P(U)$ with $\bar{s}_{1} \leqslant y_{1} \leqslant \underline{s}_{1}$. In particular, $\sigma(x)$ is constant for $\bar{r}_{1} \leqslant x_{1} \leqslant \underline{r}_{1}$, which implies that the line segments $c[x]$ for $x \in[\bar{r}, \underline{r}]$ are parallel; but this means that they must be on the same straight line $m$ through $\bar{s}$ and $\underline{s}$. Let $\bar{y}$ be the upper endpoint of $c[\bar{r}]$ and let $\underline{y}$ be the lower endpoint of $c[\underline{r}]$. Then $[\bar{y}, \underline{y}] \subseteq P(U), \bar{y}_{1}=\bar{r}_{1}$ and $\underline{y}_{2}=\underline{r}_{2}$. See, again, Figure 3 for an illustration. Similarly, let $\ell$ be the straight line through $\bar{r}$ and $\underline{r}$, then $[\bar{x}, \underline{x}] \subseteq A P(U)$, where $\bar{x}$ is the point of $\ell$ with $\bar{x}_{2}=\bar{s}_{2}$ and $\underline{x}$ is the point of $m$ with $\underline{x}_{1}=\underline{s}_{1}$. Now it is straightforward to check that $[\bar{x}, \underline{x}]$ and $[\bar{y}, \underline{y}]$ satisfy the conditions in the theorem. 